\title{
Role of exosomes and exosomal microRNAs in hepatocellular carcinoma: Potential in diagnosis and antitumour treatments (Review)
}

\author{
JING-HUA PAN ${ }^{1}$, HONG ZHOU $^{2}$, XIAO-XU ZHAO $^{1}$, HUI DING $^{1}$, WEI LI $^{1}$, LI QIN $^{3}$ and YUN-LONG PAN ${ }^{1}$ \\ Departments of ${ }^{1}$ General Surgery and ${ }^{2}$ Gynecology, The First Affiliated Hospital of Jinan University; \\ ${ }^{3}$ Department of Histology and Embryology, Medical School of Jinan University, Guangzhou, Guangdong 510632, P.R. China
}

Received December 28, 2016; Accepted January 3, 2018

DOI: $10.3892 /$ ijmm.2018.3383

\begin{abstract}
Communication between hepatocellular carcinoma (HCC) cells and their environment is essential for the development and progression of HCC. Exosomes, which are microvesicles secreted by a number of cell types, are carriers of intercellular information and regulate the tumour microenvironment. Studies have demonstrated that exosomes are involved in the communication between HCC cells, endothelial cells and stem cells, and that they serve important roles in the metastasis and invasion, immune evasion and immunotherapy of HCC. In addition, the mechanism of HCC-derived exosome-mediated microRNA (miRNA) transfer is important in the environmental modulation of HCC growth and progression. As exosomes can be used for detecting and monitoring HCC, they can potentially serve as specific biomarkers for early-stage tumours and the tumour metastasis of HCC. Moreover, mesenchymal stem cell-derived exosomes can be transfected with miRNAs to inhibit HCC development. Therefore, as nucleic acid delivery vehicles, exosomes show a tremendous potential for effective treatment against HCC. In the present review, recent advances in our understanding of the source, composition and function of exosomes in $\mathrm{HCC}$, and their potential value in the early diagnosis and treatment of HCC, are summarized.
\end{abstract}

\section{Contents}

1. Introduction

2. Source, composition and function of exosomes

3. Role of exosomes in tumour initiation and progression

4. Role of exosomes in the occurrence and development of HCC

5. Applications of exosomes in $\mathrm{HCC}$

6. Conclusion and future directions

Correspondence to: Professor Yun-long Pan, Department of General Surgery, The First Affiliated Hospital of Jinan University, 613 HuangPu Da Dao, Guangzhou, Guangdong 510632, P.R. China E-mail: tpanyl@jnu.edu.cn

Key words: hepatocellular carcinoma, exosome, microvesicles, tumour microenvironment, biomarker, microRNA

\section{Introduction}

Primary liver cancer (PLC) is one of the most common malignant tumours, and hepatocellular carcinoma (HCC) is the most frequently occurring primary liver malignancy and a leading cause of cancer-associated mortality globally (1). Despite advances in prevention, screening, and novel diagnostic and therapeutic technologies, the incidence and mortality rates have continued to rise, and the 5-year survival rate of patients with $\mathrm{HCC}$ is $<20 \%(2,3)$. Therefore, the early diagnosis and treatment of liver cancer is of particular importance. At present, the serum $\alpha$-fetoprotein (AFP) test is a common and important early diagnostic method of liver cancer, but this biomarker has a low specificity (4). Determining the molecular mechanisms of the proliferation and metastasis of liver cancer, and identifying more specific biomarkers to prevent it, is vital to improve the survival rate for patients.

Exosomes are important as substance transport carriers for biological information exchange and in the regulation of the cellular microenvironment via the delivery of a range of biological molecules, including proteins, lipids and nucleic acids. Tumour cell-derived exosomes are involved in intercellular communication, tumour angiogenesis, tumour metastasis, and drug and radiotherapy resistance (5-7). Studies have shown that cancer cells release a high level of exosomes and that the exosome components vary in different pathological and physiological conditions $(8,9)$. The contents of exosomes are precisely adjusted in tumour cells, which suggests that the exosomes may serve an important role in the process of the tumour formation and development (10). Therefore, the contents of exosomes appear to be specific diagnostic biomarkers for early-stage tumours and tumour metastasis (11). In the present review, recent progress in our understanding of the biological mechanisms, diagnosis and treatments of exosomes in HCC are summarized.

\section{Source, composition and function of exosomes}

Exosomes are membranous vesicles released into the extracellular space by cells after fusion of intracellular multivesicular bodies to the cytomembrane (12). The existence of such vesicles had been previously observed $(13,14)$, but they were termed 
exosomes by Johnstone et al in 1987 (15). These nanoparticles contain a membrane lipid bilayer and have a cup-like shape with diameters of between 30 and $150 \mathrm{~nm}$ under the electron microscope (16). Exosomes are found in the majority of, if not all, biological fluids, including urine, plasma, saliva, bronchial lavage fluid, breast milk, cerebrospinal fluid, amniotic fluid, abdominal cavity effusion and cell culture supernatant (17-19). A variety of cells can secrete exosomes, including B lymphocytes, T cells, mast cells, dendritic cells, tumour cells, endothelial cells and mesenchymal stem cells (20).

Exosomes are rich in content, and exosomes from different sources have been found to contain 9,769 types of proteins, 3,408 types of mRNAs and 2,838 types of microRNAs (miRNAs/miRs), according to the latest exosome database (http://www.exocarta.org/). The proteins and RNAs in exosomes are expressed at different levels in different diseases and physiological conditions (10). More importantly, the expression of certain proteins and RNAs in the exosomes is specific to certain tissues and cell types (20). Additionally, exosomes contain a variety of lipid molecules that can not only participate in a variety of biological processes, but also serve an important role in the morphological stability of exosomes in the extracellular fluid, protecting their contents from degradation by extracellular enzymes (21). Accordingly, it can be hypothesized that the level of exosomes has great clinical potential as a non-invasive diagnostic method (22).

Exosomes exist in the body fluids and tissues, suggesting that they may be involved in various physiological or pathological processes. Exosomes convey information by means of their vesicle contents and are considered to be the third type of signalling mechanism between cells, which is as important as cell contact-dependent signal transduction and signalling transduction mediated by soluble molecules (23). Under physiological conditions, the contents of exosomes are precisely regulated by donor cells and reflect donor cell function. To exchange and transmit biological information between cells, the donor cell transfers genetic materials to target cells through the 'transportation' function of the exosomes (24). Under pathological conditions, the diseased cells can also transfer their contents, such as viruses and miRNAs, to normal cells, and cause the normal cells to be become infected and cytopathic (25), or transfer oncogenes to normal cells, leading to tumour invasion and metastasis (26).

\section{Role of exosomes in tumour initiation and progression}

Exosomes serve a dual role in tumour initiation and progression. On the one hand, the exosomes of normal cells can inhibit the proliferation of tumour cells by transferring tumour suppressor genes into the cancerous cells, allowing the tumour suppressor genes to block the corresponding signalling pathways $(27,28)$. The exosomes of tumour cells can also induce specific antitumour effects. For example, dendritic cells have been shown to induce potent cluster of differentiation (CD) $8^{+}$ T cell-dependent antitumour effects, suggesting that exosomes are relevant for immuno-interventions (29). On the other hand, exosomes serve an important role in the tumoural process and have the ability to promote the occurrence, development and metastasis of tumours. The exosomes of cancerous cells, which can inhibit natural killer cells and cytotoxic T cells, promote tumour growth (30); they can transfer the genetic material of cancerous cells to normal cells, triggering the uninhibited growth and differentiation of normal cells, which could be one of the mechanisms of tumour invasion. Exosomes can also be transported by the blood and body fluids to other tissues and organs (31). Therefore, exosomes are an important component of the tumour microenvironment, and are involved in cell signal transduction and the process of tumour formation and degradation. Tumour exosomes can assist in deciphering the process of tumour formation and metastasis, and provide novel approaches for the clinical diagnosis and treatment of tumours.

\section{Role of exosomes in the occurrence and development of HCC}

Role of exosome miRNAs in HCC. Several studies (32-34) have shown that specific miRNAs are associated with the occurrence and development of liver cancer. It has been demonstrated that the expression of miR-21, miR-221 and miR-222 is increased in liver cancer tissue compared with that in normal liver tissue (35-37). By contrast, the expression of miR-122-a, miR-145, miR-199-a and miR-223 is decreased (34,38-40). In addition, miR-338 is closely associated with the characteristics of liver cancer, including tumour size, differentiation, vascular invasion and intrahepatic metastasis (41). miR-221, miR-103, let-7a, miR-181c, miR-181a and miR-26a (RG-6) are stably expressed in serum samples of HCC patients, and Fornari et al (42) also identified that an exosomal secretion of miR-519d, miR-21, miR-221 and miR-1228 was present in patients with HCC, together with an association between tissue and circulating levels of miR-519d, miR-494 and miR-21. Thus, miRNAs are important in the progression of liver cancer (Fig. 1) (43). With in-depth research on the transportation function of these vesicles, exosomal miRNAs have become a focus of attention. Exosomal miRNAs derived from HCC are summarized in Table I.

A large variation in miRNA expression levels exists between donor cells and their autocrine or paracrine exosomes. In Hep3B-derived exosomes, a total of 134 types of miRNAs have been identified. Of these, 55 were differentially expressed by $>4$-fold in exosomes compared with their donor cells; 25 of these miRNAs were enriched (up to 166-fold), and 30 miRNAs were depleted (up to 113-fold). It is particularly important to note that 11 types of miRNAs are only expressed in exosomes (20). In another liver cancer cell line, SMMC-7721, exosomes were found in the cell culture supernatant. Compared with that in the donor cells, the miR-423-5p and miR-21-5p levels in the exosomes were not significantly different, whereas let-7d-5p, let-7b-5p and let-7c-5p exhibited lower levels of expression, and miR-486-5p and miR-10b-5p exhibited higher levels of expression (44). Therefore, donor cells can determine the specific miRNAs loaded into the exosomes to achieve their functions.

miRNAs can be transferred between cells via exosomes and can affect target cells. For example, exosomes can transfer miR-122 between Huh7 and HepG2 human liver cancer cell lines. Huh7 cell-derived exosomes transfer miR-122 to HepG2 cells, which can inhibit the growth of HepG2 cells and hasten the ageing process of these cells (45). This transmission can also occur between different source cells; the exosomes derived from adipose mesenchymal stem cells can transfer 
Table I. miRNAs in exosomes of HCC.

\begin{tabular}{|c|c|c|c|c|c|}
\hline First author/s & Year & Country & $\begin{array}{l}\text { Source of } \\
\text { exosomes }\end{array}$ & miRNAs & (Ref.) \\
\hline Kogure et al & 2011 & USA & $\mathrm{CCS}$ & $\begin{array}{l}\text { miR-584, miR-517c, miR-378, miR-520f, } \\
\text { miR-142-5p, miR-451, miR-518d, miR-215, } \\
\text { miR-376a, miR-133b and miR-367 }\end{array}$ & $(20)$ \\
\hline Chiba et al & 2012 & Japan & CCS & miR-21, miR-192 and miR-221 & $(31)$ \\
\hline $\begin{array}{l}\text { Basu and } \\
\text { Bhattacharyya }\end{array}$ & 2014 & India & $\mathrm{CCS}$ & $\operatorname{miR}-122$ & $(45)$ \\
\hline Wang et al & 2014 & China & Serum & $\operatorname{miR}-21$ & (19) \\
\hline Wei et al & 2015 & China & $\mathrm{CCS}$ & $\begin{array}{l}\operatorname{miR}-423-5 p, \operatorname{miR}-21-5 \text {, plet-7d-5p, let-7b-5p, } \\
\text { let-7c-5p, miR-486-5p and miR-10b-5p }\end{array}$ & $(44)$ \\
\hline Sugimachi et al & 2015 & Japan & Serum & miR-718 and miR-1246 & $(48)$ \\
\hline Liu et al & 2015 & China & Rabbit serum & miR-10b, miR-21, miR-122 and miR-200a & $(58)$ \\
\hline Li et al & 2015 & China & Serum & miR-10b, miR-21, miR-122 and miR-200a & (59) \\
\hline Chen et al & 2015 & USA & CCS & $\operatorname{miR}-122$ & $(53)$ \\
\hline Sohn et al & 2015 & Korea & Serum & $\begin{array}{l}\text { miR-18a, miR-221, miR-222, miR-224, miR101, } \\
\text { miR-106b, miR-122, miR-195, miR-21 and miR-93 }\end{array}$ & $(63)$ \\
\hline Fornari et al & 2015 & Italy & $\mathrm{CCS}$ & miR-519d, miR-21, miR-221 and miR-1228 & $(42)$ \\
\hline Lou et al & 2015 & China & CCS & $\operatorname{miR}-122$ & $(46)$ \\
\hline Li et al & 2015 & China & Serum & $\begin{array}{l}\text { miR-221, miR-103, let-7a, miR-181c, } \\
\text { miR-181a and miR-26a }\end{array}$ & $(43)$ \\
\hline
\end{tabular}



Figure 1. Composition and miRNAs in the HCC-derived exosome. The figure shows the double membrane structure of the specific intracellular miRNAs of HCC-derived exosomes. The transmembrane proteins and cytosolic proteins with membrane binding capacity of the exosome can be used as markers for isolation and identification. HCC, hepatocellular carcinoma; miR/miRNA, microRNA; LncRNA, long non-coding RNAs; HSP, heat shock protein; LBPA, lactoferrin binding protein A; PD-1L, programmed death-ligand 1; LAMP, lysosomal-associated membrane protein; MHC, major histocompatibility complex; GAPDH, glyceraldehyde-3-phosphate dehydrogenase; Tsg101, tumor susceptibility gene 101; ICAM, intercellular adhesion molecule.

miR-122 to HepG2 cells (46). In human and mouse liver cells and primary B cells, exosomes can shuttle between different cells and transfer endogenous miRNAs (47). Chiba et al (31) found that colon cancer cell-derived exosomes transferred miR-21, miR-192 and miR-221 to the liver cancer HepG2 cell line and the lung cancer A549 cell line. 
Exosomes can also transport miRNAs to target cells and then act on the corresponding target genes to alter their functions. Lou et al (46) found that miR-122 could be transported to HCC cells via exosomes, which negatively regulated the target genes of miR-122 and improved the sensitivity of the HCC cells to chemotherapy drugs. Additionally, exosomal miR-718 can regulate the target gene homeobox B8 to inhibit the differentiation of liver HCC cells. Patients with low levels of serum-derived exosome miR-718 showed more tumour recurrence after liver transplantation (48). Therefore, exosomes can transfer miRNAs between cells, and these miRNAs regulate gene expression in the target cells and serve important roles in tumour invasion, metastasis and drug resistance.

Exosome-mediated immune escape of HCC. Viral components can be assembled into exosomes. Exosomes derived from $B$ cells infected with Epstein-Barr (EB) virus can be detected by the miRNAs of EB virus (49). With regard to liver cancer, persistent infection with hepatitis virus is one of the most important factors in liver tumourigenesis. There is considerable evidence to suggest that soluble immunoregulatory molecules in the exosomes secreted by HepG2 cells significantly inhibit lymphocyte proliferation (50). Recent evidence indicates that hepatitis A virus (HAV) can be protected from antibody-mediated neutralization by exosomes (51). In addition, hepatitis $\mathrm{C}$ virus (HCV) released from donor cells by way of exosomes can infect other hepatocytes. One study reported that HCV-infected Huh7.5.1 liver cancer cells can transport HCV via exosomes, and that $\mathrm{HCV}$ then escapes immunological surveillance and infects intact Huh7.5.1 cells (52). Another study showed that exosomes also mediated persistent HCV infection caused by autophagy (25), and a low Rab27a level resulted in decreased HCV RNA and protein levels in virus-infected cells (53). Thus, exosomes can mediate the escape of hepatitis virus from the human immune system, resulting in persistent infection.

Exosome-mediated HCC metastasis and invasion. Exosomes are a unique and important mechanism for signal transduction between liver cancer cells and target cells. For example, the paracrine or autocrine exosomes of liver cancer cells are rich in small RNAs and proteins, and these RNAs and proteins can be transferred by exosomes to promote HCC cell metastasis (20). Due to the natural transportation effect of the exosome, RNAs carried by exosomes can reach and impact distant cells and produce different cell phenotypes, and this may be an important mechanism of the distant metastasis of liver cancer (54). For example, HCC cells pack selective miRNAs into exosomes by means of ceramide. These exosomes can regulate the expression and downstream signalling pathways of transforming growth factor- $\beta$-activated kinase 1 of target cells, and regulate the growth and apoptosis of transformed cells (20).

In addition, certain RNAs can be transferred to other cells and tissues by means of exosomes and build a suitable microenvironment for tumour cell metastasis. A recent study suggested that in $\mathrm{CD} 90^{+}$liver cancer stem cells associated with the metastasis and recurrence of liver cancer, exosomes derived from $\mathrm{CD} 90^{+} \mathrm{Huh} 7$ cells could induce effects on tube formation and cell-cell adhesion of human umbilical vein endothelial cells (HUVECs). CD90 ${ }^{+}$cells express the long non-coding RNA (lncRNA) H19 and release it via exosomes that are able to enter endothelial cells and transfer lncRNA H19 into the corresponding target cells, which upregulate the synthesis and release of vascular endothelial growth factor to stimulate angiogenesis and promote adhesion between CD90 + Huh7 cells and endothelial cells (26).

In addition to small RNAs, exosomes can also transfer associated proteins to promote the invasion and metastasis of liver cancer. For example, the vasorin protein secreted by HCC HepG2 cells can be transferred to HUVECs by exosomes and enhance the migration of HUVECs (55). Accumulating evidence $(10,32,41)$ suggests that exosomes from HCC cell lines with a high metastatic potential are rich in cancer-promoting mRNAs and proteins, such as proto-oncogene Met, S100 family members and the caveolins. One previous study further showed that the uptake of these shuttled molecules triggered phosphoinositide 3-kinase/AKT and mitogen-activated protein kinase signalling pathways in MIHA (an immortalized hepatocyte line), with increased secretion of active matrix metalloproteinase-2 (MMP-2) and MMP-9, which enhanced the invasion and metastasis of HCC cells (56).

On the other hand, the exosomes from other cancer cells can also enhance the process of invasion and metastasis. A recent study demonstrated that exosomes derived from SW480 colorectal cancer cells induced the phosphorylation of extracellular signal-regulated kinase $1 / 2$ following their uptake into HepG2 cells and promoted recipient HepG2 cell migration (57). Therefore, in the microenvironment of liver cancer, the exosomes can be used to disseminate cancer genes and proteins, and they serve an important role in the process of the invasion and metastasis of liver cancer.

\section{Applications of exosomes in HCC}

Exosomes as diagnostic markers. Exosomes carry specific biomarkers derived from tumour cells, and the concentration of their contents is associated with the invasive ability of the tumour cells and the tumour microenvironment. Therefore, the basic information on the cancer cells can be obtained by analysis of the composition of exosomes, which suggests that the exosomes can serve as a tool for cancer diagnosis (Fig. 2) (58). Owing to the protective lipid membrane of exosomes, their contents of DNA, RNA and protein cannot easily be degraded, which renders fresh and preserved samples available for analysis. More importantly, exosomes can be obtained from numerous body fluids, which makes detection of exosomes a promising method for tumour diagnosis and treatment, and possibly ideal for the method of 'liquid biopsy' (59). Based on the analysis of a large number of serum samples, previous studies have found that the level of glypican-1-positive exosomes in the serum of pancreatic cancer patients is significantly higher than that in healthy individuals. A further study indicating that patients with early pancreatic cancer have a higher abundance of glypican-1-positive exosomes in the serum than healthy subjects provided an important basis for the application of exosomes to the early diagnosis of pancreatic cancer (60). In addition, evidence indicates that miR-21 serves an important role in tumourigenesis and tumour development. As the miR-21 serum content of tumour patients is extremely low, previous studies had mainly focused on the detection of miR-21 expression level in the cancer tissue, which greatly limited the clinical utility of miR-21 as a diagnostic tumour 


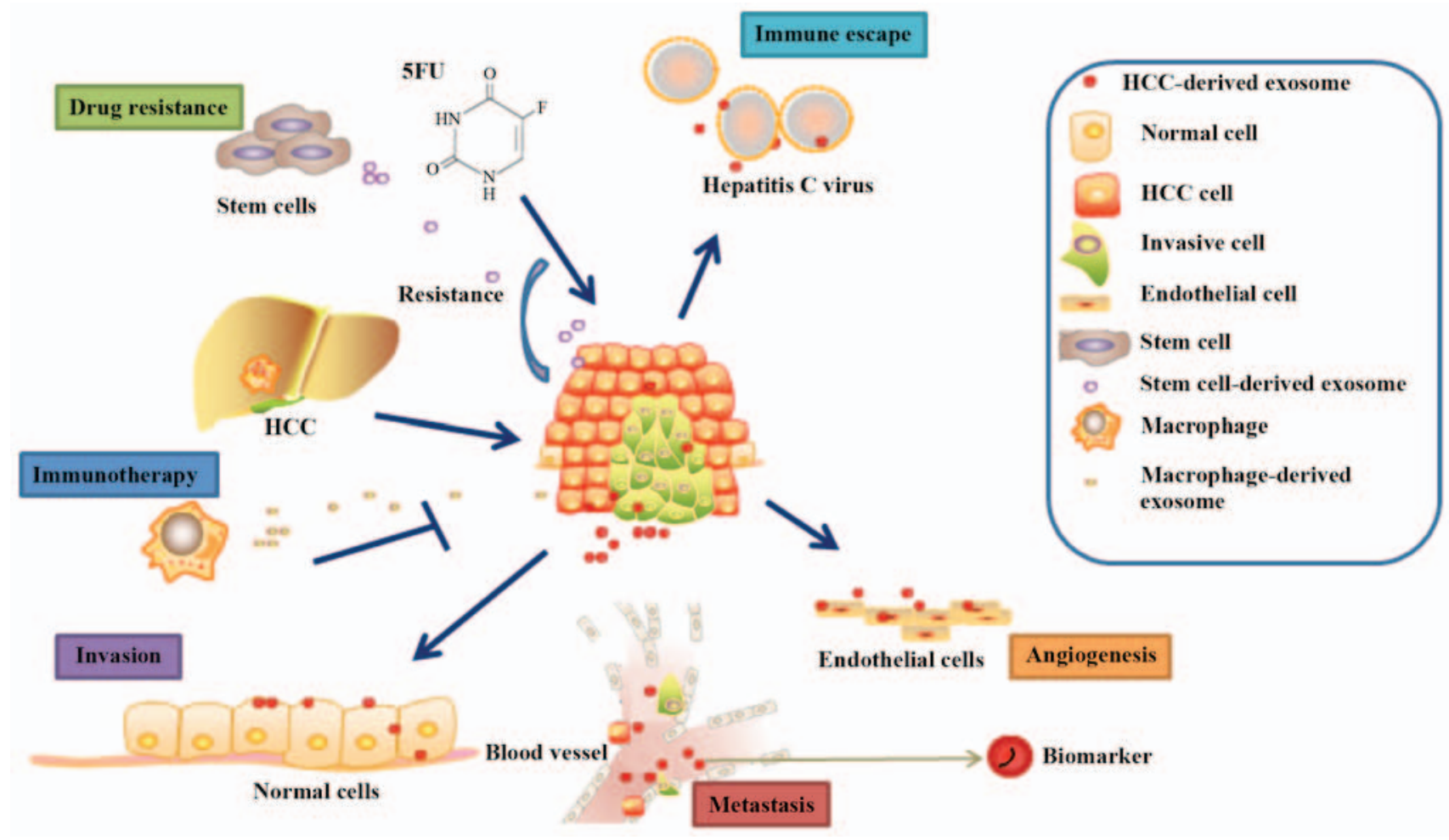

Figure 2. Function of exosomes in HCC. The figure shows the activity of HCC-derived exosomes, including immune escape, angiogenesis, metastasis and invasion, as well as their potential use as biomarkers. The exosomes from other sources, including stem cells and macrophages, transfer to HCC and perform functions such as drug resistance and immunotherapy. HCC, hepatocellular carcinoma; 5FU, 5-fluorouracil

marker (61). A previous study (62) found that the miR-21 level in serum-derived exosomes increased significantly in patients with cancer, particularly in those with malignant glioma and oesophageal squamous carcinoma, compared with that in a healthy group, which suggests that exosomal miRNAs would likely become a marker for cancer diagnosis and treatment.

miR-21 can also be detected in the serum of patients with liver cancer and chronic hepatitis B (CHB). Compared with the CHB group and healthy individuals, the level of miR-21 expression in serum-derived exosomes of patients with liver cancer increased significantly and was associated with liver cirrhosis and liver cancer staging. More importantly, the abundance of exosomal miR-21 is significantly higher than that in whole serum, which indicates that exosomal miR-21 would be a more sensitive diagnostic marker (19). Other exosomal miRNAs may also be diagnostic markers of liver cancer. For example, in the serum of patients with HCC, the exosomal contents of miR-18a, miR-221, miR-222 and miR-224 were found to be significantly higher than those in hepatitis and liver cirrhosis groups, whereas the expression levels of miR-101, miR-106b, miR-122 and miR-195 were significantly reduced (63). For the identification of specific miRNAs in exosomes from the serum of patients with recurrent HCC, Sugimachi et al (48) compared the microarray-based expression profiling of miRs derived from exosomes in the serum of patients with and without HCC recurrence and found that decreased expression of miR-718 in the serum exosomes of HCC patients was associated with HCC tumour aggressiveness. However, the different expression of these miRNAs in serum exosomes remains controversial. For example, Wang et al (19) found that miR-21 was more easily detected in serum-derived exosomes than in complete serum of HCC patients. Compared with that in a CHB group and a healthy group, the miR-21 levels in serum-derived exosomes were significantly increased (30 samples per group). However, Sohn et al (63) found no significant difference in the level of miR-21 expression in the serum of HCC patients or liver cirrhosis patients (30 samples per group). It was hypothesized that the reported differences may be associated with the sample selection, the size of the sample and the appraisal method of the miRNAs.

TUC339 is an lncRNA that is highly enriched within extracellular vesicles (EVs) released from HCC-derived tumour cells (64). This lncRNA has been implicated in modulating tumour cell growth and adhesion. The emerging data on lncRNAs indicate the presence of several tumour-associated IncRNAs, certain of which have been functionally linked to processes involved in tumour growth. The integrity and the functional role of tumour-specific lncRNAs within EVs have yet to be established, and their presence or enrichment within tumour cell-derived EVs suggests their potential as HCC biomarkers (65).

Role of exosomes in chemotherapy drug resistance of HCC. The development (46) of drug resistance is the main cause of failure of cancer chemotherapy. Liver cancer patients easily develop resistance to conventional chemotherapy drugs (Fig. 2), such as 5-fluorouracil and doxorubicin. Therefore, it is urgent to improve the efficacy of liver cancer chemotherapy. miR-122, whose expression is significantly lower in HCC compared with normal liver tissue, serves multiple roles in the physiological and pathological processes of HCC (66). Recent evidence indicates that miR-122 can alter the chemotherapeutic sensitivity of $\mathrm{HCC}$ cells by downregulating drug resistance-related genes, including multidrug resistance gene-1, glutathione $\mathrm{S}$ 
transferase- $\pi$ and multidrug resistance-associated protein, and by regulating the expression of apoptosis-related genes, such as Bcl-2-like 2, and cell cycle-related genes, such as cyclin B1.

The adipose tissue-derived mesenchymal stem cell (AMSC) can also produce a large number of exosomes. Lou et al (46) found that AMSCs transfected with miR-122 can effectively assemble miR-122 into AMSC-secreted exosomes, which can then be transported into HCC cells and promote cell apoptosis and cell cycle arrest. miR-122 can negatively regulate the expression of its target genes, such as cyclin B1 and insulin-like growth factor 1 receptor, which may improve the sensitivity of HCC cells to chemotherapeutic drugs.

The exosomes of tumour cells are rich in heat shock protein (HSP) and common tumour antigen, which can be used as cell tumour vaccines, but the efficacy of these vaccines has been found to be limited (67). One study (68) reported that exosome-immune mice can induce the response of cytotoxic T lymphocyte (CTLs) against targeted HCC cells. Following combined treatment with exosomes and cisplatin, HCC cells showed enhanced susceptibility to CTLs, which significantly prolonged the survival time of the mice. To further investigate this mechanism (68), it was hypothesized that following immunization of mice with exosomes, FasL is expressed on spleen lymphocytes and binds to the Fas on the surface of target cells, which induces tumour cell apoptosis. The results indicate that exosomes can synergize with chemotherapeutic drugs and significantly enhance the antineoplastic effect of these drugs. On the other hand, exosomes can also induce drug resistance in HCC. Isolated exosomes from two invasive HCC cell lines were able to induce sorafenib resistance in vitro by the activation of the hepatocyte growth factor/c-Met/Akt signalling pathway and the inhibition of sorafenib-induced apoptosis. Sorafenib resistance was also induced in vivo by the inhibition of sorafenib-induced apoptosis. Furthermore, exosomes derived from highly invasive tumour cells exhibited greater efficacy compared with exosomes derived from less invasive cells (69). The aforementioned studies have shown the important role of exosomes in the drug resistance of liver cancer cells, thus indicating a novel strategy for improving the effectiveness of chemotherapy when treating HCC.

Exosomes and immunotherapy for HCC. The shuttle of exosome-bound proteins and genetic material from one cell type to another suggests the feasibility of HCC immunotherapy. For example, miR-142 and miR-223 can be transferred from human macrophages to liver cells by exosomes and inhibit the proliferation or growth of tumour cells (27). A previous study showed that bone marrow mesenchymal stem cells developed significant antitumour activity following sensitization with HCC cell-derived exosomes and inhibited the proliferation of HCC cells (Fig. 2). These results suggested that sensitization with HCC cell-derived exosomes may be a novel type of antitumour therapy (70). In addition, the immunogenicity of tumour cells can be enhanced by stimulating exosomes to secrete and express their contents. Drugs such as 5-Aza-2'-deoxycytidine (5-Aza-CdR) can directly inhibit DNA methylation and increase the corresponding gene expression. There is considerable evidence to suggest that 5-Aza-CdR can improve the antitumour immune response. Subsequent to treatment with 5-Aza-CdR, the levels of HSP70, human leucocyte antigen-I (HLA-I) and cancer/testis antigen 1 proteins were increased in exosomes produced by
HCC cells, which provides evidence for 5-Aza-CdR-modified exosome-based anti-HCC immunotherapy (71). MS-275, the histone deacetylase enzyme inhibitor, had a similar effect by promoting the release of HSP70, HLA-I and CD80 by HCC HepG2 and Hep3B cells (72). Anticancer drugs can also promote the production and release by HepG2 cells of more exosomes carrying HSPs; these exosomes can induce HSP-specific natural killer (NK) cell activity, which has higher immunogenicity. In addition, adipose-derived mesenchymal stem cell-derived exosomes promoted $\mathrm{NK}$ and T-cell antitumour responses in rats, thereby facilitating HCC suppression associated with an early apparent increase of the diffusion coefficient and low-grade tumour differentiation (73). However, there has been no report of the application of exosomes to the immunotherapy of HCC patients, and further studies are required.

\section{Conclusion and future directions}

Exosomes are a novel class of intercellular signal mediators that exhibit involvement in a number of pathological conditions, including HCC. The present review summarized the roles and probable mechanisms of exosomes in $\mathrm{HCC}$, and showed the potential clinical applications of exosomes in the detection and treatment of the disease. However, the detailed mechanisms of exosomes in the invasion and metastasis of HCC are not fully understood, which hinders their application in the diagnosis and treatment of HCC. Future studies should focus on determining their mechanisms and identifying potential diagnostic and therapeutic strategies in HCC.

Multiple histopathological changes occur in HCC, including angiogenesis and increased vascular permeability, which are associated with tumourigenesis, progression, invasion and chemoresistance. Previous studies have shown that tumour exosome-secreted miR-105 destroys vascular endothelial barriers to promote metastasis in early-stage breast cancer (74). The high level of expression of Vasorin in HCC cell exosomes could affect the migration of endothelial cells (56). Close connections exist between endothelial cells, pericytes and exosomes. As HCC-derived exosomes are known to serve important roles in the tumour microenvironment, greater knowledge of the role of $\mathrm{HCC}$-derived exosomes in angiogenesis, vascular function and structure will enhance our understanding of anti-angiogenesis therapy and possibly improve the efficiency of chemotherapy and radiation treatment.

Standard technology must be established as soon as possible for the purification and isolation of exosomes. As the extracellular milieu is complex, it is not easy to achieve separation of non-vesicular entities from exosomes (75). A minimal set of biochemical, biophysical and functional standards is provided by the International Society for Extracellular Vesicles, which should be used for the attribution of any specific biological cargo or functions to extracellular vesicles (76). However, the heterogeneity in size and the probable subpopulations of exosomes (77) suggest that further improvement of purification and characterization is required to obtain pure preparations and easily identify exosomes of interest.

With regard to clinical applications, exosomes have the potential to be used for the diagnosis and antitumour treatments of HCC. Clinical trials have been performed to improve the utilization by antigen-presenting activity and enhance the antitumour 
immune response $(78,79)$, but their safety and efficacy require further confirmation. Therefore, a deeper understanding of exosomes in HCC should bring breakthroughs and transformative changes in the diagnosis and treatment of $\mathrm{HCC}$ in the future.

\section{Acknowledgements}

This study was supported by grants from the National Natural Science Foundation of China (no. 81472849), Guangdong Natural Science Research (no. 2014A030313383) and the Guangdong High-level University Construction Fund for Jinan University (no. 88016013034).

\section{Competing interests}

The authors declare that they have no competing interests.

\section{References}

1. Torre LA, Bray F, Siegel RL, Ferlay J, Lortet-Tieulent J and Jemal A: Global cancer statistics, 2012. CA Cancer J Clin 65 87-108, 2015.

2. El-Serag HB and Rudolph KL: Hepatocellular carcinoma: Epidemiology and molecular carcinogenesis. Gastroenterology 132: 2557-2576, 2007.

3. El-Serag HB: Hepatocellular carcinoma. N Engl J Med 365: 1118-1127, 2011.

4. Jeon Y, Jang ES, Choi YS, Kim JW and Jeong SH: Glypican-3 level assessed by the enzyme-linked immunosorbent assay is inferior to alpha-fetoprotein level for hepatocellular carcinoma diagnosis. Clin Mol Hepatol 22: 359-365, 2016

5. Milane L, Singh A, Mattheolabakis G, Suresh M and Amiji MM Exosome mediated communication within the tumor microenvironment. J Control Release 219: 278-294, 2015.

6. Yu DD, Wu Y, Shen HY, Lv MM, Chen WX, Zhang XH, Zhong SL, Tang JH and Zhao JH: Exosomes in development, metastasis and drug resistance of breast cancer. Cancer Sci 106: 959-964, 2015

7. Tang Y, Cui Y, Li Z, Jiao Z, Zhang Y, He Y, Chen G, Zhou Q, Wang W, Zhou X, et al: Radiation-induced miR-208a increases the proliferation and radioresistance by targeting p21 in human lung cancer cells. J Exp Clin Cancer Res 35: 7, 2016.

8. Soung YH, Nguyen T, Cao H, Lee J and Chung J: Emerging roles of exosomes in cancer invasion and metastasis. BMB Rep 49 $18-25,2016$.

9. Riches A, Campbell E, Borger E and Powis S: Regulation of exosome release from mammary epithelial and breast cancer cells - a new regulatory pathway. Eur J Cancer 50: 1025-1034, 2014.

10. Yang N, Li S, Li G, Zhang S, Tang X, Ni S, Jian X, Xu C, Zhu J and Lu M: The role of extracellular vesicles in mediating progression, metastasis and potential treatment of hepatocellular carcinoma. Oncotarget 8: 3683-3695, 2017.

11. Wang Z, Chen JQ, Liu JL and Tian L: Exosomes in tumor microenvironment: Novel transporters and biomarkers. J Trans Med 14: 297, 2016

12. Théry C, Zitvogel L and Amigorena S: Exosomes: Composition, biogenesis and function. Nat Rev Immunol 2: 569-579, 2002.

13. Wolf P: The nature and significance of platelet products in human plasma. Br J Haematol 13: 269-288, 1967.

14. Harding C, Heuser J and Stahl P: Receptor-mediated endocytosis of transferrin and recycling of the transferrin receptor in rat reticulocytes. J Cell Biol 97: 329-339, 1983.

15. Johnstone RM, Adam M, Hammond JR, Orr L and Turbide C: Vesicle formation during reticulocyte maturation. Association of plasma membrane activities with released vesicles (exosomes) J Biol Chem 262: 9412-9420, 1987.

16. Mathivanan S, Ji H and Simpson RJ: Exosomes: Extracellular organelles important in intercellular communication. J Proteomics 73: 1907-1920, 2010.

17. Oosthuyzen W, Sime NE, Ivy JR, Turtle EJ, Street JM, Pound J, Bath LE, Webb DJ, Gregory CD, Bailey MA, et al: Quantification of human urinary exosomes by nanoparticle tracking analysis. J Physiol 591: 5833-5842, 2013.

18. Street JM, Barran PE, Mackay CL, Weidt S, Balmforth C, Walsh TS, Chalmers RT, Webb DJ and Dear JW: Identification and proteomic profiling of exosomes in human cerebrospinal fluid. J Transl Med 10: 5, 2012.
19. Wang H, Hou L, Li A, Duan Y, Gao H and Song X: Expression of serum exosomal microRNA-21 in human hepatocellular carcinoma. Biomed Res Int 2014: 864894, 2014.

20. Kogure T, Lin WL, Yan IK, Braconi C and Patel T: Intercellular nanovesicle-mediated microRNA transfer: A mechanism of environmental modulation of hepatocellular cancer cell growth. Hepatology 54: 1237-1248, 2011.

21. Yuyama K, Sun H, Mitsutake S and Igarashi Y: Sphingolipid-modulated exosome secretion promotes clearance of amyloid- $\beta$ by microglia. J Biol Chem 287: 10977-10989, 2012.

22. Herreros-Villanueva M and Bujanda L: Glypican-1 in exosomes as biomarker for early detection of pancreatic cancer. Ann Transl Med 4: 64, 2016

23. Ludwig AK and Giebel B: Exosomes: Small vesicles participating in intercellular communication. Int J Biochem Cell Biol 44: 11-15, 2012.

24. Tkach $\mathrm{M}$ and Théry $\mathrm{C}$ : Communication by extracellular vesicles: Where we are and where we need to go. Cell 164: 1226-1232, 2016.

25. Shrivastava S, Devhare P, Sujijantarat N, Steele R, Kwon YC, Ray R and Ray RB: Knockdown of autophagy inhibits infectious hepatitis $C$ virus release by the exosomal pathway. J Virol 90: 1387-1396, 2015.

26. Conigliaro A, Costa V, Lo Dico A, Saieva L, Buccheri S, Dieli F, Manno M, Raccosta S, Mancone C, Tripodi M, et al: CD90+ liver cancer cells modulate endothelial cell phenotype through the release of exosomes containing H19 lncRNA. Mol Cancer 14: $155,2015$.

27. Aucher A, Rudnicka D and Davis DM: MicroRNAs transfer from human macrophages to hepato-carcinoma cells and inhibit proliferation. J Immunol 191: 6250-6260, 2013.

28. Putz U, Howitt J, Doan A, Goh CP, Low LH, Silke J and Tan SS: The tumor suppressor PTEN is exported in exosomes and has phosphatase activity in recipient cells. Sci Signal 5: ra70, 2012.

29. Azmi AS, Bao B and Sarkar FH: Exosomes in cancer development, metastasis, and drug resistance: A comprehensive review. Cancer Metastasis Rev 32: 623-642, 2013.

30. Whiteside TL: Immune modulation of T-cell and NK (natural killer) cell activities by TEXs (tumour-derived exosomes). Biochem Soc Trans 41: 245-251, 2013

31. Chiba M, Kimura M and Asari S: Exosomes secreted from human colorectal cancer cell lines contain mRNAs, microRNAs and natural antisense RNAs, that can transfer into the human hepatoma HepG2 and lung cancer A549 cell lines. Oncol Rep 28: $1551-1558,2012$

32. Xu Y, Xia F, Ma L, Shan J, Shen J, Yang Z, Liu J, Cui Y, Bian X, Bie P, et al: MicroRNA-122 sensitizes HCC cancer cells to adriamycin and vincristine through modulating expression of MDR and inducing cell cycle arrest. Cancer Lett 310: 160-169, 2011.

33. Wang B, Majumder S, Nuovo G, Kutay H, Volinia S, Patel T, Schmittgen TD, Croce C, Ghoshal K and Jacob ST: Role of microRNA-155 at early stages of hepatocarcinogenesis induced by choline-deficient and amino acid-defined diet in C57BL/6 mice. Hepatology 50: 1152-1161, 2009.

34. Li S, Fu H, Wang Y, Tie Y, Xing R, Zhu J, Sun Z, Wei L and Zheng X: MicroRNA-101 regulates expression of the v-fos FBJ murine osteosarcoma viral oncogene homolog (FOS) oncogene in human hepatocellular carcinoma. Hepatology 49: 1194-1202, 2009.

35. Meng F, Henson R, Wehbe-Janek H, Ghoshal K, Jacob ST and Patel T: MicroRNA-21 regulates expression of the PTEN tumor suppressor gene in human hepatocellular cancer. Gastroenterology 133: 647-658, 2007.

36. Varnholt H, Drebber U, Schulze F, Wedemeyer I, Schirmacher P, Dienes HP and Odenthal M: MicroRNA gene expression profile of hepatitis $\mathrm{C}$ virus-associated hepatocellular carcinoma. Hepatology 47: 1223-1232, 2008.

37. Yang YF, Wang F, Xiao JJ, Song Y, Zhao YY, Cao Y, Bei YH and Yang CQ: MiR-222 overexpression promotes proliferation of human hepatocellular carcinoma HepG2 cells by downregulating p27. Int J Clin Exp Med 7: 893-902, 2014.

38. Gramantieri L, Ferracin M, Fornari F, Veronese A, Sabbioni S, Liu CG, Calin GA, Giovannini C, Ferrazzi E, Grazi GL, et al: Cyclin G1 is a target of miR-122a, a microRNA frequently downregulated in human hepatocellular carcinoma. Cancer Res 67: 6092-6099, 2007.

39. Wang G, Zhu S, Gu Y, Chen Q, Liu X and Fu H: MicroRNA-145 and microRNA-133a inhibited proliferation, migration, and invasion, while promoted apoptosis in hepatocellular carcinoma cells via targeting FSCN1. Dig Dis Sci 60: 3044-3052, 2015. 
40. Wang Q, Yu X, Li Q, Qin L, Tan S, Zeng X, Qiu X, Tang B, Jin J, Liao W, et al: Association between miR-199a rs74723057 and MET rs1621 polymorphisms and the risk of hepatocellular carcinoma. Oncotarget 7: 79365-79371, 2016

41. Huang XH, Wang Q, Chen JS, Fu XH, Chen XL, Chen LZ, Li W, Bi J, Zhang LJ, Fu Q, et al: Bead-based microarray analysis of microRNA expression in hepatocellular carcinoma: miR-338 is downregulated. Hepatol Res 39: 786-794, 2009.

42. Fornari F, Ferracin M, Trerè D, Milazzo M, Marinelli S, Galassi M, Venerandi L, Pollutri D, Patrizi C, Borghi A, et al: Circulating microRNAs, miR-939, miR-595, miR-519d and miR-494, identify cirrhotic patients with HCC. PLoS One 10: e0141448, 2015.

43. Li Y, Zhang L, Liu F, Xiang G, Jiang D and Pu X: Identification of endogenous controls for analyzing serum exosomal miRNA in patients with hepatitis B or hepatocellular carcinoma. Dis Markers 2015: 893594, 2015.

44. Wei JX, Lv LH, Wan YL, Cao Y,Li GL, Lin HM,Zhou R, Shang CZ, $\mathrm{Cao} \mathrm{J}, \mathrm{He} \mathrm{H}$, et al: Vps4A functions as a tumor suppressor by regulating the secretion and uptake of exosomal microRNAs in human hepatoma cells. Hepatology 61: 1284-1294, 2015.

45. Basu S and Bhattacharyya SN: Insulin-like growth factor-1 prevents miR-122 production in neighbouring cells to curtail its intercellular transfer to ensure proliferation of human hepatoma cells. Nucleic Acids Res 42: 7170-7185, 2014

46. Lou G, Song X, Yang F, Wu S, Wang J, Chen Z and Liu Y: Exosomes derived from miR-122-modified adipose tissue-derived MSCs increase chemosensitivity of hepatocellular carcinoma. J Hematol Oncol 8: 122, 2015.

47. Pan Q, Ramakrishnaiah V, Henry S, Fouraschen S, de Ruiter PE, Kwekkeboom J, Tilanus HW, Janssen HL and van der Laan LJ: Hepatic cell-to-cell transmission of small silencing RNA can extend the therapeutic reach of RNA interference (RNAi) Gut 61: 1330-1339, 2012.

48. Sugimachi K, Matsumura T, Hirata H, Uchi R, Ueda M, Ueo H, Shinden Y, Iguchi T, Eguchi H, Shirabe K, et al: Identification of a bona fide microRNA biomarker in serum exosomes that predicts hepatocellular carcinoma recurrence after liver transplantation. Br J Cancer 112: 532-538, 2015.

49. Pegtel DM, Cosmopoulos K, Thorley-Lawson DA, van Eijndhoven MA, Hopmans ES, Lindenberg JL, de Gruijl TD, Würdinger T and Middeldorp JM: Functional delivery of viral miRNAs via exosomes. Proc Natl Acad Sci USA 107: 6328-6333, 2010.

50. Xiao W, Dong W, Zhang C, Saren G, Geng P, Zhao H, Li Q, Zhu J, $\mathrm{Li} \mathrm{G}$, Zhang S, et al: Effects of the epigenetic drug MS-275 on the release and function of exosome-related immune molecules in hepatocellular carcinoma cells. Eur J Med Res 18: 61, 2013.

51. Feng Z, Hensley L, McKnight KL, Hu F, Madden V, Ping L, Jeong SH, Walker C, Lanford RE and Lemon SM $\neq$ : A pathogenic picornavirus acquires an envelope by hijacking cellular membranes. Nature 496: 367-371, 2013

52. Ramakrishnaiah V, Thumann C, Fofana I, Habersetzer F, Pan Q, de Ruiter PE, Willemsen R, Demmers JA, Stalin Raj V, Jenster $\mathrm{G}$, et al: Exosome-mediated transmission of hepatitis $\mathrm{C}$ virus between human hepatoma Huh7.5 cells. Proc Natl Acad Sci USA 110: 13109-13113, 2013.

53. Chen TC, Hsieh CH and Sarnow P: Supporting role for GTPase Rab27a in hepatitis C virus RNA replication through a novel miR-122-mediated effect. PLoS Pathog 11: e1005116, 2015.

54. Tickner JA, Urquhart AJ, Stephenson SA, Richard DJ and O'Byrne KJ: Functions and therapeutic roles of exosomes in cancer. Front Oncol 4: 127, 2014.

55. Huang A, Dong J, Li S, Wang C, Ding H, Li H, Su X, Ge X, Sun L, Bai C, et al: Exosomal transfer of vasorin expressed in hepatocellular carcinoma cells promotes migration of human umbilical vein endothelial cells. Int J Biol Sci 11: 961-969, 2015.

56. He M, Qin H, Poon TC, Sze SC, Ding X, Co NN, Ngai SM, Chan TF and Wong N: Hepatocellular carcinoma-derived exosomes promote motility of immortalized hepatocyte through transfer of oncogenic proteins and RNAs. Carcinogenesis 36: 1008-1018, 2015.

57. Chiba M, Watanabe N, Watanabe M, Sakamoto M, Sato A, Fujisaki M, Kubota S, Monzen S, Maruyama A, Nanashima N, et al: Exosomes derived from SW480 colorectal cancer cells promote cell migration in HepG2 hepatocellular cancer cells via the mitogen-activated protein kinase pathway. Int J Oncol 48: 305-312, 2016

58. Liu WH, Ren LN, Wang X, Wang T, Zhang N, Gao Y, Luo H, Navarro-Alvarez $\mathrm{N}$ and Tang LJ: Combination of exosomes and circulating microRNAs may serve as a promising tumor marker complementary to alpha-fetoprotein for early-stage hepatocellular carcinoma diagnosis in rats. J Cancer Res Clin Oncol 141: 1767-1778, 2015.
59. Li Y, Xiang GM, Liu LL, Liu C, Liu F, Jiang DN and Pu XY: Assessment of endogenous reference gene suitability for serum exosomal microRNA expression analysis in liver carcinoma resection studies. Mol Med Rep 12: 4683-4691, 2015.

60. Melo SA, Luecke LB, Kahlert C, Fernandez AF, Gammon ST, Kaye J, LeBleu VS, Mittendorf EA, Weitz J, Rahbari N, et al: Glypican-1 identifies cancer exosomes and detects early pancreatic cancer. Nature 523: 177-182, 2015.

61. Skog J, Würdinger T, van Rijn S, Meijer DH, Gainche L, Sena-Esteves M, Curry WT Jr, Carter BS, Krichevsky AM and Breakefield XO: Glioblastoma microvesicles transport RNA and proteins that promote tumour growth and provide diagnostic biomarkers. Nat Cell Biol 10: 1470-1476, 2008.

62. Tanaka Y, Kamohara H, Kinoshita K, Kurashige J, Ishimoto T, Iwatsuki M, Watanabe $\mathrm{M}$ and Baba $\mathrm{H}$ : Clinical impact of serum exosomal microRNA-21 as a clinical biomarker in human esophageal squamous cell carcinoma. Cancer 119: 1159-1167, 2013

63. Sohn W, Kim J, Kang SH, Yang SR, Cho JY, Cho HC, Shim SG and Paik YH: Serum exosomal microRNAs as novel biomarkers for hepatocellular carcinoma. Exp Mol Med 47: e184, 2015.

64. Kogure T, Yan IK, Lin WL and Patel T: Extracellular vesicle-mediated transfer of a novel long noncoding RNA TUC339: A mechanism of intercellular signaling in human hepatocellular cancer. Genes Cancer 4: 261-272, 2013.

65. Mohankumar S and Patel T: Extracellular vesicle long noncoding RNA as potential biomarkers of liver cancer. Brief Funct Genomics 15: 249-256, 2016.

66. Bandiera S, Pfeffer S, Baumert TF and Zeisel MB: miR-122 - a key factor and therapeutic target in liver disease. J Hepatol 62: 448-457, 2015

67. Mou DL, Jia ZS and Bai XF: Exosome: Trojan horse in immunotherapy. Sheng Li Ke Xue Jin Zhan 36: 113-118, 2005 (In Chinese).

68. Wang SH, Shen Y, Li J, Xiang ZW, Fan WK and Chen L: Experimental studies on anti-mouse hepatocellular carcinoma effects of cisplatin combined with exosomes. Xi Bao Yu Fen Zi Mian Yi Xue Za Zhi 25: 49-52, 2009 (In Chinese).

69. Qu Z, Wu J, Wu J, Luo D, Jiang C and Ding Y: Exosomes derived from HCC cells induce sorafenib resistance in hepatocellular carcinoma both in vivo and in vitro. J Exp Clin Cancer Res 35: 159,2016

70. Ma B, Jiang H, Jia J, Di L, Song G, Yu J, Zhu Y, Lu Z, Wang X, Zhou X, et al: Murine bone marrow stromal cells pulsed with homologous tumor-derived exosomes inhibit proliferation of liver cancer cells. Clin Transl Oncol 14: 764-773, 2012.

71. Xiao WH, Sanren GW, Zhu JH, Li QW, Kang HR, Wang RL, Song LP and Ye M: Effect of 5-aza-2'-deoxycytidine on immune-associated proteins in exosomes from hepatoma. World J Gastroenterol 16: 2371-2377, 2010.

72. Li QW, Xiao WH, Sarengaowa G, Dong WW, Zhao HX, Duan XY, Zhu JH, Kang HR, Fu Y, Hao YX, et al: Histone deacetylase inhibitor MS-275 treatment alters immune molecule content and categories in hepatocarcinoma exosomes. Zhonghua Gan Zang Bing Za Zhi 20: 231-235, 2012 (In Chinese).

73. Ko SF, Yip HK, Zhen YY, Lee CC, Lee CC, Huang CC, Ng SH and Lin JW: Adipose-derived mesenchymal stem cell exosomes suppress hepatocellular carcinoma growth in a rat model: Apparent diffusion coefficient, natural killer T-cell responses, and histopathological features. Stem Cells Int 2015: 853506, 2015.

74. Zhou W, Fong MY, Min Y, Somlo G, Liu L, Palomares MR, Yu Y, Chow A, O'Connor ST, Chin AR, et al: Cancer-secreted miR-105 destroys vascular endothelial barriers to promote metastasis. Cancer Cell 25: 501-515, 2014.

75. Mincheva-Nilsson L, Baranov V, Nagaeva $\mathrm{O}$ and Dehlin E: Isolation and characterization of exosomes from cultures of tissue explants and cell lines. Curr Protoc Immunol 115: 14.42.1-14.42.21, 2016.

76. Lötvall J, Hill AF, Hochberg F, Buzás EI, Di Vizio D, Gardiner C, Gho YS, Kurochkin IV, Mathivanan S, Quesenberry P, et al: Minimal experimental requirements for definition of extracellular vesicles and their functions: A position statement from the International Society for Extracellular Vesicles. J Extracell Vesicles 3: 26913, 2014.

77. Bobrie A and Théry C: Exosomes and communication between tumours and the immune system: Are all exosomes equal? Biochem Soc Trans 41: 263-267, 2013.

78. Viaud S, Ploix S, Lapierre V, Théry C, Commere PH, Tramalloni D, Gorrichon K, Virault-Rocroy P, Tursz T, Lantz O, et al: Updated technology to produce highly immunogenic dendritic cell-derived exosomes of clinical grade: a critical role of interferon-gamma. J Immunother 34: 65-75, 2011.

79. Dai S, Wei D, Wu Z, Zhou X, Wei X, Huang H and Li G: Phase clinical trial of autologous ascites-derived exosomes combined with GM-CSF for colorectal cancer. Mol Ther 16: 782-790, 2008.

This work is licensed under a Creative Commons Attribution-NonCommercial-NoDerivatives 4.0 International (CC BY-NC-ND 4.0) License. 